> Les récentes mesures promulguées par le NIH aux États-Unis, appuyées par des membres de l'université de Harvard, exigeant la mise à disposition publique par les chercheurs d'une version électronique de leurs publications, marquent un tournant dans l'attitude des chercheurs et des institutions vis-à-vis de l'accès libre (Open Access) et des « archives ouvertes ». Cette législation, acquise en dépit de l'opposition des grandes maisons d'édition, a contribué à faire connaître le mouvement Open Access et laisse présager son amplification. Cet article analyse l'histoire du mouvement Open Access, ses récentes évolutions aux États-Unis, au Canada et en Europe, et les raisons de sa légitimité. Cette politique de renforcement de l'Open Access ne fait que témoigner de l'intégration de la publication comme élément indissociable de la démarche de recherche. II paraîtrait donc judicieux de considérer que le coût de la publication scientifique fait partie du coût de la recherche scientifique. II n'en constitue d'ailleurs qu'une fraction minuscule, de l'ordre de $1 \%$. II semblerait dès lors cohérent de simplement ajouter le coût de la publication scientifique à celui de la recherche. Ainsi, le financement de l'évaluation par les pairs, de la correction de la langue et de la mise en ligne des articles sur des serveurs adéquats serait couvert, et l'accès libre aux résultats de la recherche assuré. <

\section{Repenser \\ le sens de \\ la communication scientifique : I'accès libre}

Jean-Claude Guédon

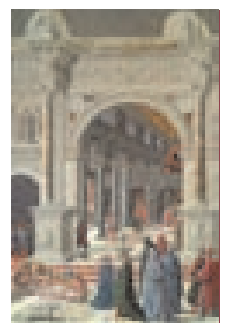

Département de littérature comparée,

Université de Montréal,

CP 6128, Succursale

Centre-ville, Montréal,

Québec H3C 3J7, Canada.

jean.claude.guedon@

umontreal.ca

une fois qu'ils auront été évalués par les pairs, et dès qu'ils seront prêts à la publication. Le public devra pouvoir consulter ces documents au plus tard 12 mois après la date officielle de publication. Il est entendu que le NIH appliquera cette politique d'accès public en conformité avec la loi du copyright ${ }^{1} \gg$.

Pour obtenir ces quelques lignes de législation, il aura fallu de nombreuses années de batailles ardues contre divers groupes de pression, en particulier contre les grandes maisons d'édition internationales soucieuses de maintenir la haute main sur l'édition scientifique et les profits qui accompagnent cette prérogative ${ }^{2}$. Pour beaucoup, la mesure promulguée par le NIH constitue une victoire décisive, d'abord parce qu'elle confirme des mesures analogues déjà adoptées par d'autres grands organismes subventionnaires en sciences biomédicales, ensuite parce que cette victoire, compte tenu de l'importance même du NIH au sein du monde des recherches biomédicales, ne peut qu'encourager des organismes analogues à prendre des décisions analogues. Bref, cette victoire, dans le cas du NIH, laisse présager une amplification du mouvement en faveur de l'accès libre aux publications scientifiques et savantes. Cette amplification s'est vue confirmée de façon spectaculaire le 12 février 2008 : les professeurs de la

${ }^{1}$ Le texte de la loi de 613 pages se trouve à l'adresse suivante: http://frwebgate. access.gpo.gov/cgi-bin/getdoc.cgi?dbname=110_cong_bills\&docid=f:h2764enr.txt. pdf. Section 218. Notre traduction.

${ }^{2}$ Peter Suber a publié une chronologie de la «saga» conduisant à la loi H.R.2764. Voir http://www.earlham.edu/ peters/fos/newsletter/08-02-07.htm\#nih. 
Faculté des Arts et des Sciences de l'Université Harvard, par un vote unanime, ont décidé de rendre obligatoire la mise à disposition publique des articles émanant de leurs recherches ${ }^{3}$. Cette résolution marque clairement un tournant dans l'attitude des chercheurs et donne une signification nouvelle au mouvement en faveur du libre accès, mouvement qui, jusqu'à maintenant, n'avait retenu l'attention que d'une minorité de chercheurs.

Mais d'où vient ce mouvement? Que signifie-t-il ? Pourquoi faut-il le soutenir?

\section{La communication entre scientifiques et ses distorsions}

La Révolution scientifique du XVII ${ }^{\mathrm{e}}$ siècle s'est inscrite sous le signe d'un dialogue indéfiniment ouvert. Chaque résultat de recherche, lorsqu'il est publié après avoir été évalué par des pairs, entre dans l'archive des sciences par le biais de la publication. Depuis 1665, date de création des Philosophical Transactions of the Royal Society de Londres, et du Journal des Sçavans de Paris, ce dialogue s'est approprié l'imprimerie et a transposé la formule de la gazette pour diffuser ses propres nouvelles, à cette différence près que, dans ce dernier cas, le vieillissement de la nouvelle est lié à son statut plutôt qu'à son âge. La nouvelle scientifique obéit aux règles d'une jurisprudence et non d'une obsolescence: son âge compte beaucoup moins que sa valeur scientifique.

Au sein de la grande conversation scientifique, les vecteurs de diffusion des nouvelles jouent un rôle évidemment essentiel. Faute d'un accès large aux périodiques scientifiques ou savants, le chercheur est fortement handicapé dans ses activités, autant pour se tenir à jour que pour éviter de dupliquer des travaux antérieurs. Corrélativement, les responsables des revues scientifiques occupent un rôle particulier dans la hiérarchie scientifique: «Gatekeepers », tel Saint-Pierre à l'entrée du paradis, ils détiennent le pouvoir de donner accès ou non à une publication dotée d'un certain niveau de prestige et de visibilité. En gérant le processus de l'évaluation par les pairs, ces «Gatekeepers » disposent d'un pouvoir d'autant plus important dans leur communauté de recherche, parfois décrite sous le nom de « collège invisible » par les sociologues, qu'il est occulté. À noter au passage que la nomination au poste de responsable de revue (directeurs, éditeur, rédacteur en chef) peut être largement influencée par une maison d'édition lorsqu'elle est propriétaire de la revue. On découvre ainsi une frange d'activités où la hiérarchie scientifique et même la substance du discours scientifique dépendent en partie du monde commercial ${ }^{4}$.

Longtemps dominées par les académies et par les sociétés savantes ou les associations de scientifiques, les revues scientifiques ont vu

\footnotetext{
${ }^{3}$ Le texte exact de la motion se trouve à l'adresse suivante: http://www.fas.harvard.edu/ secfas/February 2008_Agenda.pdf. La section du blog de Peter Suber correspondant à la semaine du 10 au 17 février 2008 (http://www.earlham.edu/ peters/fos/2008_02_10_fosblogarchive.html) offre l'essentiel des réactions immédiates à cette annonce. Certains la considèrent encore plus importante que celle du NIH parce qu'elle vient directement des chercheurs eux-mêmes. Une clause permet le retrait d'un article particulier mais son utilité apparaît problématique, surtout du point de vue d'une maison d'édition, puisqu'il faut répéter la procédure de requête, article par article.

${ }^{4}$ Un autre exemple de ce type d'interférence qui jette une lumière trouble sur les publications scientifiques est celui de la publicité dans les revues médicales. Frederic Crews écrit: «même les revues les plus respectées hésitent à s'opposer aux compagnies qui leur achètent de la publicité... » dans son excellent article, «Talking Back to Prozac », New York Review of Books, Vol. 54, n 19 (décembre 2007), 10-14 [notre traduction].
}

apparaître une troisième catégorie d'acteurs dont l'importance n'est devenue manifeste qu'après la Deuxième Guerre mondiale: les presses commerciales. D'abord largement nationales avec, bien entendu, une vocation internationale correspondant à l'universalité des résultats scientifiques, ces presses commerciales se sont mondialisées à partir des années 1960. Le processus de mondialisation est passé par plusieurs étapes, parmi lesquelles les suivantes paraissent essentielles ${ }^{5}$ : - Construction graduelle d'un marché inélastique ${ }^{6}$ des revues scientifiques fondé en grande partie sur le succès du «Science Citation Index 》 (SCl) imaginé par Eugene Garfield.

- Développement d'une première maison d'édition visant directement un marché mondial plutôt qu'un marché national complété par des ventes à l'étranger: il s'agit de l'entreprise «Pergamon Science», lancée par Robert Maxwell ${ }^{7}$, qui repose en particulier sur une réforme de l'évaluation par les pairs. Pour mieux contrôler le marché inélastique défini par le $\mathrm{SCI}$, Maxwell tente également de s'approprier ce dernier.

- Augmentation rapide du prix des périodiques scientifiques, en particulier à partir de la fin des années 1970. Ceci engendre le mouvement que les bibliothécaires américains appellent la «Serial Pricing Crisis». Certaines grandes sociétés scientifiques, en particulier l'American Chemical Society, s'engouffrent dans l'espace de prix ainsi créé pour financer leurs autres activités et deviennent ainsi en partie prisonnières de leurs publications pour maintenir l'équilibre de leur budget. - En parallèle, les maisons d'édition commerciales s'engagent dans un processus accéléré de fusions dans le but de créer des collections toujours plus importantes de revues et ainsi de mieux concurrencer leurs adversaires. Ainsi, Elsevier rachète Pergamon Science au printemps de 1991 pour 400 millions de $\mathrm{f}$.

${ }^{5}$ Nous schématisons ici l'argument que nous avons développé dans «In Oldenburg's Long Shadow: Librarians, Research Scientists, Publishers, and the Control of Scientific Publishing » (2001), disponible en ligne à l'URL suivant : http://www.arl. org/resources/pubs/mmproceedings/138guedon.shtml.

${ }^{6}$ Rappelons qu'un marché inélastique est un marché où l'augmentation du prix influe peu sur la demande, parce que la marchandise demandée est indispensable. En l'absence de contrôles étatiques, cette situation conduit généralement à des surprofits. La «Serial pricing crisis » en constitue un exemple frappant.

${ }^{7}$ Traditionnellement, les revues scientifiques et savantes tendaient à fonctionner par articles recommandés et les membres du comité éditorial décidaient du sort de ces articles. Le tout conduisait à une situation de cooptation où les jeunes en particulier étaient graduellement introduits par leurs aînés dans les échanges scientifiques, le tout généralement sur une base nationale. Maxwell a introduit l'habitude d'envoyer les articles à des arbitres extérieurs aux comités éditoriaux et ainsi souligner l'objectivité et la transparence de l'évaluation par les pairs. Par exemple, J. F. A. Sprent, rédacteur-en-chef de International Journal for Parasitology, une publication émanant de l'Australie, mais mondialisée par Pergamon Press, explique que "Contributions from one country are always reviewed by referees from another country». J. F. A. Sprent, «Editorial in Celebration of the $65^{\text {th }}$ Birthday of Robert Maxwell and the $40^{\text {th }}$ Anniversary of Pergamon Press », International Journal for Parasitology, vol. 18, $n^{\circ} 4$ (1988), 419. 
- Le passage au numérique conduit à progressivement délaisser la vente de numéros de revues pour vendre des licences d'accès à cellesci. Jouant de leur poids, les grands éditeurs commerciaux offrent alors des «big deals » aux bibliothèques, siphonnant ainsi une fraction croissante des budgets d'acquisition de celles-ci. Or, ces mêmes budgets stagnent au cours de cette période, ce qui place les bibliothèques dans une situation de plus en plus difficile. Par ailleurs, la même tendance fragilise les petits éditeurs, ainsi que les petites sociétés scientifiques qui doivent souvent se résoudre à vendre leurs revues aux grosses maisons internationales.

- Le résultat général de cette tendance s'exprime par la définition d'un ensemble particulier de revues - les revues dites «centrales »- dont le nombre est artificiellement limité. Par ce moyen, le reste des publications scientifiques est rejeté dans les limbes d'une quasi invisibilité. On parle souvent d'environ 20000 à 24000 revues scientifiques «qui comptent», publiant environ 2 millions d'articles par an. Cette collection de revues dessine ainsi les contours d'un cercle mi-élitiste, mi-oligarchique selon le point de vue adopté. Certains analystes proches de grandes maisons d'édition, tel Michael Mabe, évaluent même le nombre de revues importantes à seulement 14000 titres environ. Or, et la coïncidence mérite d'être soulignée, ce nombre se rapproche considérablement de la couverture de SCOPUS, le nouveau rival du SCl. Scopus, rappelons-le, est chapeauté par ReedElsevier qui reprend ainsi l'ambition de Maxwell de contrôler un outil essentiel d'évaluation des revues, à commencer par les siennes ${ }^{8}$.

- La mondialisation des publications scientifiques a donc conduit à un vaste mouvement de concentration de la publication scientifique, concentration qui ressemble de plus en plus à un cartel, avec tous les effets délétères que ce genre de structure d'entreprise engendre. En plus de prix élevés, cette situation conduit à d'énormes inégalités (pour ne pas dire injustices) d'accès qui jouent bien évidemment en faveur des pays riches, et, à l'intérieur de ceux-ci, en faveur des institutions les plus riches. En effet, lorsque la bibliothèque est bien financée, le chercheur qui est en fait un lecteur subventionné, peut entretenir la douce illusion d'un accès libre à la littérature scientifique. Dirigées très majoritairement par des scientifiques des pays riches, poursuivant les orientations et intérêts de recherche des savants de ces pays, ces revues disposent d'un potentiel collectif de contrôle qui se rapproche dangereusement de la capacité d'influencer l'orientation des recherches scientifiques. Une phraséologie un peu plus hardie pourrait même suggérer la possibilité d'une sorte de politique informelle ou occulte des sciences liée à cette collection de quelques milliers de titres. L'accès à la publication ainsi qu'à l'information est donc sévèrement contraint au point de jouer le rôle de discriminant entre une «société de l'information» où les progrès des technologies de communication peuvent servir tout le monde, et une «économie de l'information» où les lois du marché assurent l'émergence d'oligarchies scientifiques susceptibles d'être au service d'intérêts certainement assez éloignés de l'universel.

${ }^{8}$ Michael Mabe, The Growth and Number of Journals, Serials, vol. 16, $n^{\circ} 2$ (juillet 2003), p. 192. Rappelons que Michael Mabe, avant de prendre la direction de STM (International Association of Scientific, Technical, and Medical Publishers), était employé par Elsevier.

\section{L'accès libre}

L'évolution de la communication scientifique a suscité une indignation croissante, d'abord parmi les bibliothécaires parce qu'ils payaient les factures, ensuite chez les chercheurs eux-mêmes. Il en est résulté une visibilité croissante du phénomène en cours et, corrélativement, la coalescence d'un mouvement en faveur de l'accès libre. Ces deux réactions ont d'abord convergé de manière ostensible, mais inefficace, dans une pétition intitulée «Public Library of Science » (PloS). Plus de trente mille scientifiques du monde entier ont signé cette pétition au cours de $2001^{9}$. La même année, une douzaine d'activistes, dont l'auteur de ces lignes, étaient invités en décembre à Budapest par l'Open Society Institute. De cette réunion est sorti le célèbre manifeste publié le 14 février 2002 et généralement connu sous le nom de Budapest Open Access Initiative ${ }^{10}$. Ce document est demeuré canonique dans le mouvement en faveur de l'accès libre, d'abord parce que c'était le premier du genre; ensuite, parce qu'il définissait deux voies pour atteindre le but désiré: d'une part, les auteurs eux-mêmes pouvaient auto-archiver leurs articles dans des dépôts institutionnels ou personnels; d'autre part, on pouvait transformer des revues scientifiques en revues en accès libre ou créer de nouvelles revues ${ }^{11}$.

Soucieuses de ne pas trop heurter de front les communautés scientifiques dont elles avaient pu sentir la colère à travers la pétition de 2001 , certaines maisons d'édition ont accepté de laisser les auteurs auto-archiver une version ou une autre de leurs articles ${ }^{12}$, offrant ainsi l'image de la conciliation tout en sachant fort bien que ces amas - le mot n'est pas trop fort - de textes numériques, même moissonnés par des moteurs de recherche spécialisés (tels OAlster ou Google Scholar) ${ }^{13}$ ne seraient pas d'une grande utilité à la majorité des chercheurs, surtout ceux qui voyaient leurs habitudes de recherche et de lecture soutenues par des bibliothèques bien nanties en documents. Par indifférence plutôt que par résistance, les chercheurs ont largement négligé l'importance de déposer leurs travaux. Les bibliothèques sont les principaux promoteurs des dépôts, mais, jusqu'à

\footnotetext{
${ }^{9}$ Le Canada a donné 623 signataires et la France 2507. Voir http://www.plos.org/ cgi-bin/plosSigned.pl.

${ }^{10}$ http://www.soros.org/openaccess/read.shtml.

${ }^{11}$ Dans le vocabulaire devenu classique du mouvement en faveur de l'accès libre, l'auto-archivage porte le nom de route «verte», tandis que les revues en accès libre renvoient à la «route d'or». Voir http://www.sherpa.ac.uk/romeo.php.

${ }^{12}$ Pour s'y retrouver dans ce dédale de politiques d'auto-archivage, une liste a été constituée, qui décrit les permissions particulières accordées par différentes maisons d'édition. Voir http://www.sherpa.ac.uk/romeo.php.

${ }^{13}$ Voir http://www.oaister.org et http://scholar.google.ca [au Canada; en France remplacer le .ca par.fr].
} 
présent, elles n'ont pas trouvé de moyens réellement efficaces pour inciter les auteurs à déposer. Les administrateurs des universités et des centres de recherche, pour leur part, n'ont pas plus compris le lien entre la pression budgétaire sur les bibliothèques et le libre accès qu'ils n'ont compris comment la façon d'évaluer les chercheurs contribuait à renforcer les structures perverses du marché des revues scientifiques. II suffit de penser au rôle incongru du facteur d'impact dans l'évaluation individuelle des performances des chercheurs pour commencer à sonder l'ineptie de ces modalités d'évaluation pourtant fort courantes ${ }^{14}$.

$\varepsilon n$ parallèle, les revues scientifiques en accès libre se sont multipliées: un outil d'indexation comme le «Directory of Open Access Journals $»^{15}$, hébergé à l'Université de Lund en Suède, recense actuellement (mars 2008) 3263 titres mais il faut bien avouer que bon nombre des «grandes» revues, celles qui jouissent d'un facteur d'impact élevé et qui, pour cette raison, comptent pour les chercheurs (en particulier pour l'évaluation de leurs prestations), ne constituent encore qu'une infime minorité de cette collection.

\section{L’obligation de dépôt}

C'est dans ce contexte que la question d'un dépôt obligatoire a commencé à voir le jour. Ce faisant, les efforts en faveur de l'accès libre ont pris un tour plus politique et une bataille sérieuse fait maintenant rage dans plusieurs pays et régions du globe autour de cette question. La loi américaine H.R. 2764 constitue un tournant majeur dans cette lutte puisque, en dépit d'efforts considérables de lobbying financés par les grandes maisons d'édition, les quelques lignes citées au début de ce texte concernent les sommes de plusieurs milliards de dollars affectées à la recherche biomédicale et environ 80000 articles par an. Des mesures semblables ont été promulguées par le Wellcome Trust en Grande-Bretagne ${ }^{16}$. Au Canada, les Instituts de recherche en santé du Canada (CIHR-IRSC) ont passé un règlement analogue:

À compter du $1^{\text {er }}$ janvier 2008 , les chercheurs qui ont obtenu de nouveaux fonds ou qui ont renouvelé leur financement auprès des IRSC doivent s'acquitter des nouvelles responsabilités suivantes:

Veiller à ce que tous les articles de recherche issus des projets financés par les IRSC soient librement accessibles sur le site Web de l'éditeur ou un dépôt en ligne dans les six mois suivant la publication ${ }^{17}$.

Le Conseil de recherche médicale de Grande-Bretagne (MRC) a adopté une loi du même genre dès le $1^{\text {er }}$ octobre 2006. Le mouvement en faveur du dépôt obligatoire s'étend donc.

En France, le mouvement en faveur des archives ouvertes a également progressé. Dès le 22 octobre 2003, I’INSERM signait la décla-

\footnotetext{
${ }^{14}$ L'expression «incongrue » peut aisément se justifier si l'on pense que le facteur d'impact a été conçu pour évaluer des revues portant sur les mêmes spécialités. Or, il est utilisé de manière routinière non seulement pour évaluer les revues en général, sans égards pour les habitudes de citations de chaque domaine, mais encore pour évaluer des individus comme si tous les auteurs rassemblés au sein d'un même périodique publiaient des articles de valeur égale.

${ }^{15}$ Voir http://www.doaj.org. Voir aussi http://www.openj-gate.com. Ce dernier site est situé en Inde et recense 4385 titres, en ajoutant des revues professionnelles aux revues strictement savantes.

${ }^{16}$ Pour une liste mondiale de politiques en matière de dépôts d'articles, consulter http://www.eprints. org/openaccess/policysignup/.

${ }^{17}$ Voir http://www.cihr-irsc.gc.ca/f/32005.html.
}

ration de Berlin sur le libre accès à la connaissance ${ }^{18}$. Depuis la fin de l'année 2005, un dépôt institutionnel HAL-INSERM recueille les publications de I'INSERM ${ }^{19}$. Malheureusement, défini seulement comme une incitation, le dépôt demeure limité. On le constate en juin 2006, lors de la rencontre des professionnels de I'IST à Nancy. ${ }^{20}$ La situation n'a pas fondamentalement changé depuis. Cela dit, l'accord entre divers organismes pour faire de HAL le dépôt institutionnel national, signé en juillet 2006, viendra à échéance en juillet 2008 : l'occasion se présentera alors certainement de reprendre la question du dépôt obligatoire et de procéder à son examen dans le sillage des décisions américaines, canadiennes et britanniques.

\section{Le rôle pionnier des sciences médicales}

On peut remarquer que les sciences médicales ont joué un rôle de premier plan dans cette tendance qui se révèle chaque jour un peu plus lourde, un peu plus irréversible. On pourrait se demander pourquoi. La réponse se trouve assez facilement. Pour l'illustrer - la place manque ici pour le démontrer - un détail et une anecdote suffiront. Le détail tourne autour de la loi américaine : si une telle loi a pu être passée dans un contexte aussi défavorable sur le plan des idéologies politiques que celui de l'administration Bush, c'est parce que chercheurs et bibliothécaires ont trouvé un allié très puissant en la personne de la «Alliance of Taxpayers for Access $»^{21}$. Cette organisation regroupant un grand nombre d'organismes de soutien pour les victimes de diverses maladies graves s'est révélée extrêmement efficace dans la lutte pour les voix des parlementaires américains. Les patients veulent simplement devenir des partenaires dans les choix offerts par la médecine et non simplement des consommateurs. Le modèle actuel de circulation des informations provenant de la recherche scientifique de pointe leur paraît trop limité, trop restrictif, surtout si l'on pense que les travaux menant aux publications scientifiques ne sont possibles que grâce aux investissements publics. Dans le contexte

\footnotetext{
${ }^{18}$ Voir http://openaccess.inist.fr/article.php3?id_article $=38 \& v^{2}=$ recherche =inserm.

${ }^{19}$ Voir http://www.hal.inserm.fr.

${ }^{20}$ On trouvera une excellente analyse de la situation française dans Dominique L'Hostis et Pascal Aventurier, «Archives ouvertes - Vers une obligation de dépôt? » (avec la collaboration de Hélène Bosc), version 2 du 5 décembre 2006, disponible en ligne à l'adresse suivante: http://www.revues.org/cost/ images/7/7f/Note-A0-version2-051206-diff-Externe.pdf. Voir aussi Nicole Pinhas, «Les archives dans le domaine bio-médical», disponible à l'adresse suivante: http://rpist.inist.fr/sites/rpist/IMG/pdf/Nicole_Pinhas.pdf. Les articles de l'Inserm sont aussi versés dans PubMed Central. See http://www.ncbi.nlm. nih.gov/sites/entrez?db=pmc\&cmd=search\&term=hal \%20inserm \%20author \%0manuscript \%5Bfilter.

${ }^{21}$ Voir http://www.taxpayeraccess.org/.
} 
du débat actuel sur les régimes d'assurance-maladie aux États-Unis et dans l'atmosphère d'une année électorale charnière, ce groupe d'électeurs ne pouvait plus être ignoré.

Dans le même ordre d'idées, prenons l'exemple, anecdotique sans doute mais éclairant néanmoins, d'un ophtalmologue français connu de nous. Cet ophtalmologue éprouve de grandes difficultés à obtenir l'accès à des articles importants pour établir le bon diagnostic dans divers cas difficiles, en dépit du fait que ce spécialiste se soit abonné individuellement à quelques revues fondamentales ${ }^{22}$. Ceci démontre que l'irrigation du réseau des praticiens en information scientifique de pointe est insuffisante. La conséquence, terrible, c'est que dans beaucoup de cas des patients sont traités avec des méthodes plus anciennes, peut-être inadéquates, voire fautives. Or, encore une fois, une bonne partie des résultats de recherches publiés est financée par des fonds publics.

Évidemment, l'argument en faveur des patients et des praticiens non rattachés à des bibliothèques universitaires se répète et s'intensifie pour le Tiers Monde ; il révèle alors une situation inadmissible, qui touche autour de 75-80\% de la population de la planète, ce qui montre que si la connaissance scientifique jouit d'une validité universelle, l'accès à cette connaissance est loin d'être aussi universel. On peut dès lors se poser une question fondamentale: qu'est-ce qui est plus important: le meilleur accès possible aux connaissances scientifique ou le maintien de profits ${ }^{23}$ pour les revues scientifiques?

Dans certains pays, comme le Brésil avec son projet SciદLO ${ }^{24}$, et au sein de certains organismes, tel le Wellcome Trust, une thèse saine se développe: elle consiste à déclarer que le coût de la publication scientifique fait partie du coût de la recherche scientifique. II n'en constitue d'ailleurs qu'une fraction minuscule, de l'ordre de $1 \%$. II semblerait dès lors cohérent de simplement ajouter le coût de la publication scientifique à celui de la recherche. Dès lors, le financement de l'évaluation par les pairs, de la correction de la langue et de la mise en ligne des articles sur des serveurs adéquats serait couvert, et l'accès libre aux résultats de la recherche assuré 25 .

\section{Conclusion}

La science, a-t-on dit, est un long dialogue indéfini et ouvert. Le grand historien des sciences, Alexandre Koyré, le définissait comme iter mentis ad veritatem. Mais ce chemin vers la vérité, il n'est plus très carrossable si partout l'on rencontre des péages élevés et si l'on se heurte à l'impossibilité d'emprunter d'autres routes, peut-être moins belles,

\footnotetext{
${ }^{22}$ Mais on apprend aussi que les abonnements individuels tendent à être traités par dessus la jambe parce qu'insignifiants sur le plan financier pour la maison d'édition, alors que ces revues peuvent coûter plusieurs centaines de dollars le titre pour un abonnement individuel annuel.

${ }^{23}$ Ces profits peuvent s'appeler «excédents» dans le cas de sociétés à but non-lucratif. L'excédent contribue alors à financer des activités, par ailleurs louables, de ces sociétés : congrès, bourses, etc. La question dans ce cas-là est un peu différente : est-il normal pour une société scientifique de soutenir des activités louables en restreignant par une barrière économique l'accès aux résultats de recherche?

${ }^{24}$ Voir http://www.scielo.org/php/index.php?lang=en.

${ }^{25}$ Ce modèle n'exclut pas la possibilité d'acheter des versions sur papier pour ceux (en nombre décroissant d'ailleurs) qui ne peuvent pas s'en passer. L'impression à la demande et tarifée répond aisément à cette objection. Ce modèle maintient aussi, bien évidemment, l'évaluation par les pairs, le contrôle de qualité éditoriale, la production effective de la revue et sa préservation. Le modèle de SciદLO au Brésil donne une première idée concrète de ce mode de fonctionnement, ce qui n'exclut pas, d'ailleurs, son perfectionnement.
}

mais qui conduisent néanmoins à la bonne destination. Dans le cas de la littérature scientifique, les voies qui se présentent prennent généralement l'allure de très belles autoroutes, extrêmement coûteuses et donc réservées à de toutes petites élites. D'ailleurs celles-ci sont entretenues dans leur élitisme en partie par ce biais. Grâce aux rapports particuliers qu'ils entretiennent avec les grands éditeurs, les scientifiques du monde entier nourrissent une conversation non pas ouverte et générale, mais restreinte à un petit nombre, empêchant ainsi une bonne partie des bénéfices de la recherche de rejaillir sur l'ensemble de la population, bien qu'il s'agisse d'activités financées par des fonds publics.

Parce que la science affiche avec raison son universalité, il semble légitime d'ouvrir les résultats de la science à tous. Un mouvement important se dessine également pour la libération des données brutes de laboratoire. Ces tendances nous rappellent que, dans un monde où la globalité des problèmes n'a d'égal que leur complexité, nous avons certainement besoin de tous les cerveaux qualifiés de la planète. Les exclure par divers moyens aussi arbitraires qu'injustes revient aussi à affaiblir nos efforts pour réduire pandémies et autre réchauffement du climat. Voilà qui devrait aussi constituer un argument fort en faveur de l'accès libre. $\diamond$

\section{SUMMARY}

\section{Reconceptualising the meaning}

of scientific communication: open access

The recent US law (H.R.2764) affecting NIH policy and the recent unanimous vote by the Arts and Science faculty of Harvard University in favour of a mandatory deposit of researchers' publications in a suitable repository have brought the Open Access movement into public light. After reviewing the historical background of Open Access, its evolution and extension in the United States, Great Britain, France and Canada are examined. Policies aiming at strengthening Open Access to scientific research are viewed as the direct consequence of treating scientific publishing as an integral part of the research cycle. It should, therefore, be wrapped into the financing of research. As the greater part of research is funded by public money, it appears legitimate to make its results as widely available as is possible. Open Access journals and repositories with strong deposit mandates form the backbone of the strategies to achieve the objective of Open Access. Despite the claims of some publishers, Open Access does not weaken or threaten the peer review process, and it does not conflict with copyright laws. $\diamond$

\section{TIRÉS À PART}

J.C. Guédon 


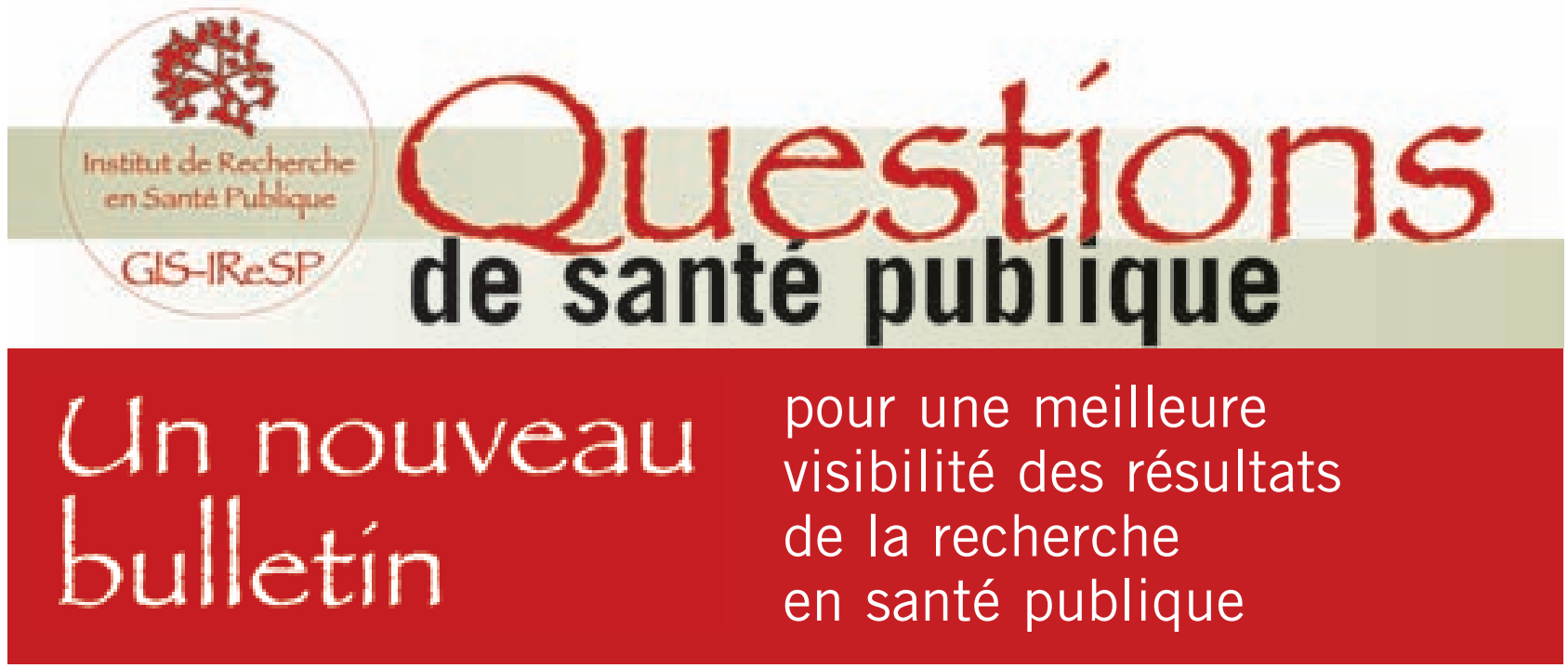

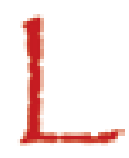

es résultats de la recherche en santé publique souffrent en France d'un réel manque de visibilité. Ceci concerne aussi bien le monde académique (hors santé publique) que le grand public et les décideurs. Pour pallier ce déficit, l'IReSP crée un bulletin à large diffusion intitulé " Questions de santé publique ", largement inspiré du bulletin mensuel d'information de I'INED «Populations et sociétés ". L'objectif éditorial est de porter à la connaissance d'un large public (enseignants, étudiants, journalistes, décideurs, milieux de la recherche, associations, public concerné) les informations les plus récentes concernant des questions importantes de santé publique, rédigées de façon facilement lisible et compréhensible pour des non spécialistes, en garantissant que les informations publiées sont validées scientifiquement. La publications concernera des faits et non des positions. Au-delà de la présentation de résultats, cette publication devrait également avoir des qualités pédagogiques, permettant au lecteur de mieux comprendre comment sont formulées et abordées les questions de santé publique et quelles sont les limites de ces études.

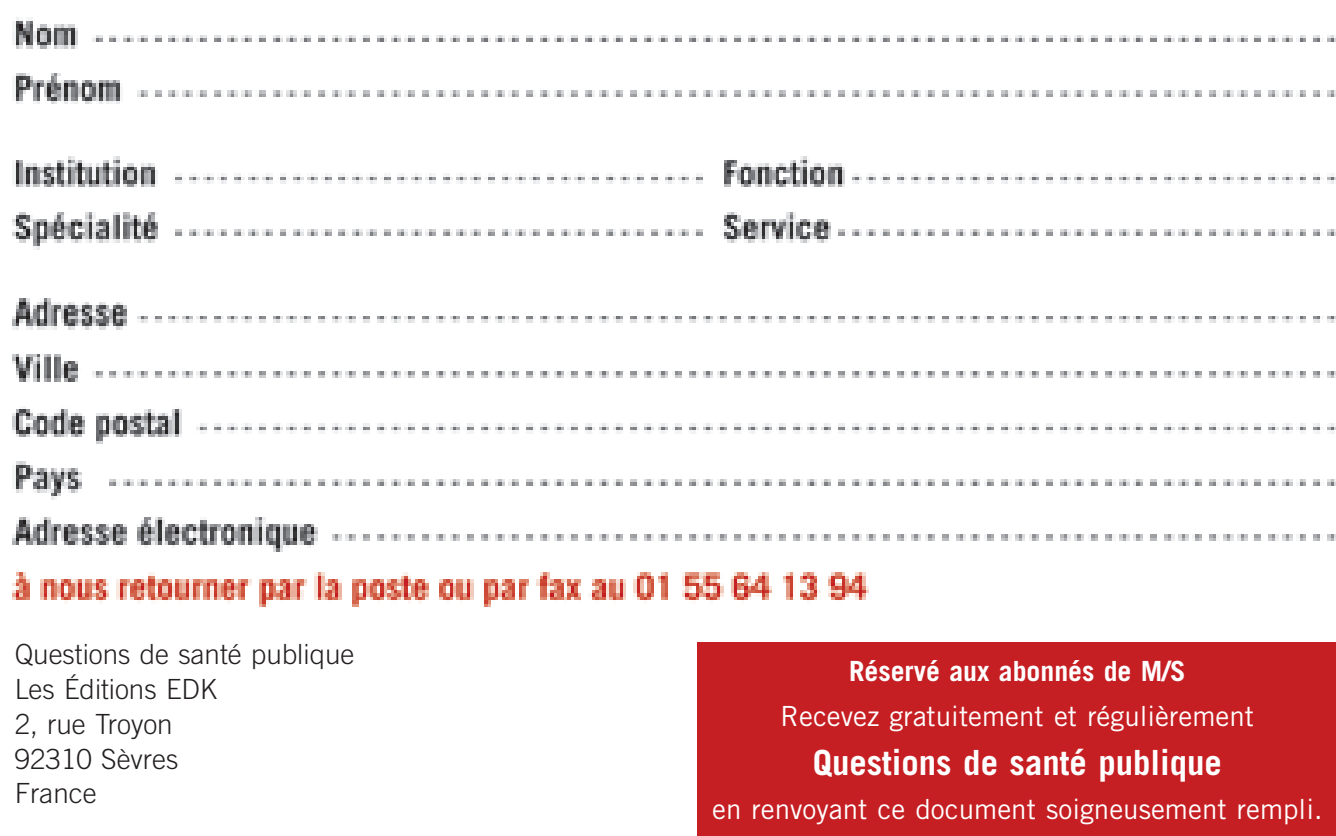

Questions de santé publique est une publication de l'Institut de Recherche en Santé Publique. I Directeur de la publication : Alfred Spira. I Rédacteur en chef : Nathalie de Parseval. I Une réalisation des Éditions EDK. 